\title{
INTERPOLATION BY COMPLETELY POSITIVE MAPS
}

\author{
CHI-KWONG LI AND YIU-TUNG POON
}

Dedicated to Professor John Conway on the occasion of his retirement.

\begin{abstract}
Given commuting families of Hermitian matrices $\left\{A_{1}, \ldots, A_{k}\right\}$ and $\left\{B_{1}, \ldots, B_{k}\right\}$, conditions for the existence of a completely positive map $\Phi$, such that $\Phi\left(A_{j}\right)=B_{j}$ for $j=$ $1, \ldots, k$, are studied. Additional properties such as unital or / and trace preserving on the map $\Phi$ are also considered. Connections of the study to dilation theory, matrix inequalities, unitary orbits, and quantum information science are mentioned.
\end{abstract}

\section{INTRODUCTION}

Denote by $M_{n, m}$ the set of $n \times m$ complex matrices, and use $M_{n}$ to denote $M_{n, n}$. Let $H_{n}$ be the set of Hermitian matrices in $M_{n}$. A matrix $A \in H_{n}$ is positive semidefinite if all eigenvalues of $A$ are nonnegative. A linear map $\Phi: M_{n} \rightarrow M_{m}$ is positive if it maps positive semidefinite matrices to positive semidefinite matrices. Suppose $M_{k}\left(M_{n}\right)$ is the algebra of block matrices of the form $\left(A_{i j}\right)_{1 \leq i, j \leq k}$ with $A_{i j} \in M_{n}$ for each pair of $(i, j)$. A linear map $\Phi: M_{n} \rightarrow M_{m}$ is completely positive if for each positive integer $k$, the map $I_{k} \otimes \Phi: M_{k}\left(M_{n}\right) \rightarrow M_{k}\left(M_{m}\right)$ defined by $\left(I_{k} \otimes \Phi\right)\left(A_{i j}\right)=\left(\Phi\left(A_{i j}\right)\right)$ is positive.

The purpose of this paper is to study the following.

Problem 1.1. Given $A_{1}, \ldots, A_{k} \in M_{n}$ and $B_{1}, \ldots, B_{k} \in M_{m}$, determine the necessary and sufficient condition for the existence of a completely positive map $\Phi: M_{n} \rightarrow M_{m}$ such that $\Phi\left(A_{j}\right)=B_{j}$ for $j=1, \ldots, k$, and possibly with the additional properties that $\Phi\left(I_{n}\right)=I_{m}$ or/and $\Phi$ is trace preserving.

Clearly, this can be viewed as an interpolation problem by completely positive maps. Denote by $H_{n}$ the set of Hermitian matrices in $M_{n}$. Since for a positive linear map $\Phi$ satisfying $\Phi(X)=Y$ if and only if $\Phi\left(X^{*}\right)=Y^{*}$ for any $(X, Y) \in M_{n} \times M_{m}$, we can focus on the study of Problem 1.1 for $\left\{A_{1}, \ldots, A_{k}\right\} \subseteq H_{n}$ and $\left\{B_{1}, \ldots, B_{k}\right\} \subseteq H_{m}$

Over half a century ago, Steinspring [19] introduced completely positive maps in the study of dilation problems for operators. Since then, the area has been studied extensively $[15,16]$. In particular, researchers have obtained interesting structure theorem for completely positive maps on matrices. For example, Choi [2] (see also [10]) showed that a linear map $\Phi: M_{n} \rightarrow M_{m}$ is

2010 Mathematics Subject Classification. Primary 14A04, 15A42,15B48, 15B51, 81P68.

Key words and phrases. Completely positive map, quantum operations, dilations, Hermitian matrices, eigenvalues, majorization. 
completely positive if and only if there exist $F_{1}, \ldots, F_{r} \in M_{n, m}$ such that

$$
\Phi(A)=\sum_{j=1}^{r} F_{j}^{*} A F_{j} .
$$

This is called an operator sum representation of the completely positive map $\Phi$.

In the context of quantum information theory, every quantum operation is a completely positive map sending quantum states to quantum states, where quantum states are represented as density matrices, i.e., positive semidefinite matrices with trace one. Because a completely positive map $\Phi: M_{n} \rightarrow M_{m}$ representing a quantum operation will send density matrices to density matrices, the map $\Phi$ is trace preserving, i.e., $\Phi(A) \in M_{m}$ and $A \in M_{n}$ always have the same trace. Therefore, in quantum information science, most studies are on trace preserving completely positive maps. On the other hand, in the $C^{*}$-algebra context, since the trace function may not be defined, most studies are on unital maps, i.e., $\Phi\left(I_{n}\right)=I_{m}$. While rich theory has been developed for completely positive maps, for example, see $[15,16]$, there are many basic problems motivated by applied topics which deserve further study.

In quantum information science, one has to study and construct quantum operations sending a specific family of density operators to another family. This clearly reduces to Problem 1.1 if we restrict our attention to density matrices $A_{1}, \ldots, A_{k} \in M_{n}$ and $B_{1}, \ldots, B_{k} \in M_{m}$; see [14].

Using the operator sum representation (1.1) of completely positive maps, and the inner product $\langle A, B\rangle=\operatorname{tr}\left(A B^{*}\right)$ for $A, B \in M_{m, n}$, one has the following result showing the connection between trace preserving completely positive maps and unital completely positive maps.

Proposition 1.2. Suppose $\Phi: M_{n} \rightarrow M_{m}$ is a completely positive map with operator sum representation in (1.1). Then $\Phi$ is unital if and only if $\sum_{j=1}^{r} F_{j}^{*} F_{j}=I_{m} ; \Phi$ is trace preserving if and only if $\sum_{i=1}^{r} F_{j} F_{j}^{*}=I_{n}$. Moreover, the dual linear map $\Phi^{*}: M_{m} \rightarrow M_{n}$ defined by

$$
\Phi^{*}(B)=\sum_{j=1}^{r} F_{j} B F_{j}^{*}
$$

is the unique linear map satisfying $\langle\Phi(A), B\rangle=\left\langle A, \Phi^{*}(B)\right\rangle$ for all $(A, B) \in M_{n} \times M_{m}$. Consequently, $\Phi$ is unital if and only if $\Phi^{*}$ is trace preserving.

The above proposition provides a link between problems and results for trace preserving completely positive maps and unital completely positive maps. Therefore, one might expect that the results and proofs for the two types of problems can be converted to each other easily. However, this does not seem to be the case as shown in our results. In fact, some results in trace preserving completely positive maps are more involved in our study, and they have no analogs for unital completely positive maps; see Remark $2.3 \mathrm{~b}$ ), and the remarks after Corollary 3.4 and Theorem 3.6.

It is known that the study of completely positive maps are closely related to the dilations of operators. Recall that a matrix $B \in M_{m}$ has a dilation $A \in M_{n}$ if there is an $n \times m$ matrix $V$ 
such that $V^{*} V=I_{m}$ and $V^{*} A V=B$. The next result shows that Problem 1.1 can be formulated as problems involving dilations and principal submatrices of a matrix.

Proposition 1.3. Suppose $\Phi: M_{n} \rightarrow M_{m}$ is a completely positive map with operator sum representation (1.1). If $F=\left[\begin{array}{c}F_{1} \\ \vdots \\ F_{r}\end{array}\right]$, then $\Phi(A)=F^{*}\left(I_{r} \otimes A\right) F$. If $\tilde{F}=\left[\begin{array}{lll}F_{1} & \cdots & F_{r}\end{array}\right]$ and $\tilde{F}^{*} A \tilde{F}=$ $\left(A_{i j}\right)$ with $A_{11}, \ldots, A_{r r} \in M_{m}$, then $\Phi(A)=A_{11}+\cdots+A_{r r}$. Furthermore, the following hold.

(a) The map $\Phi$ is unital if and only if $F^{*} F=I_{m}$.

(b) The map $\Phi$ is trace preserving if and only if $\tilde{F} \tilde{F}^{*}=I_{n}$.

Proposition 1.4. Suppose $\Phi: M_{n} \rightarrow M_{m}$ is a completely positive map. Given unitaries $U \in M_{n}$ and $V \in M_{m}$, define $\Psi: M_{n} \rightarrow M_{m}$ by $\Psi(X)=V^{*} \Phi\left(U^{*} X U\right) V$. Then $\Psi$ is also completely positive. Furthermore, $\Psi$ is unital and/or trace preserving if and only if $\Phi$ has the corresponding property. If $\Phi(X)=\sum_{j=1}^{r} F_{j}^{*} X F_{j}$, then $\Psi(X)=\sum_{j=1}^{r}\left(U F_{j} V\right)^{*} X\left(U F_{j} V\right)$.

Propositions $1.2-1.4$ will be used in the subsequent discussion. Our paper is organized as follows. In Section 2, we determine the condition for the existence of completely positive maps (possibly with additional conditions such as unital or/and trace presering) $\Phi: M_{n} \rightarrow M_{m}$ sending a given commuting family of matrices in $H_{n}$ to another one in $H_{m}$. In Section 3, we give a more detailed analysis for the case when each family has only one matrix. Some related results, additional remarks, and open problems will be mentioned in Section 4 .

\section{COMPLETELY POSITIVE MAPS BETWEen COMMUTING FAMILIES}

In this section, we consider (unital) completely positive maps $\Phi: M_{n} \rightarrow M_{m}$ sending a given commuting family of matrices in $H_{n}$ to another commuting family in $H_{m}$.

Theorem 2.1. Let $\left\{A_{1}, \ldots, A_{k}\right\} \subseteq H_{n}$ and $\left\{B_{1}, \ldots, B_{k}\right\} \subseteq H_{m}$ be two commuting families. Then there exist unitary matrices $U \in M_{n}$ and $V \in M_{m}$ such that $U^{*} A_{i} U$ and $V B_{i} V^{*}$ are diagonal matrices with diagonals $\mathbf{a}_{i}=\left(a_{i 1}, \ldots, a_{i n}\right)$ and $\mathbf{b}_{i}=\left(b_{i 1}, \ldots, b_{i m}\right)$ respectively, for $i=1, \ldots, k$. The following conditions are equivalent.

(a) There is a completely positive map $\Phi: M_{n} \rightarrow M_{m}$ such that $\Phi\left(A_{i}\right)=B_{i}$ for $i=1, \ldots, k$.

(b) There is an $n \times m$ nonnegative matrix $D=\left(d_{p q}\right)$ such that

$$
\left(b_{i j}\right)=\left(a_{i j}\right) D .
$$

Suppose (b) holds. For $1 \leq j \leq m$, let $F_{j}$ be the $n \times n$ matrix having the $j$ th column equal to $\left(\sqrt{d_{1 j}}, \sqrt{d_{2 j}}, \ldots, \sqrt{d_{n j}}\right)^{t}$ and zero elsewhere. Then we have

$$
B_{i}=\sum_{j=1}^{r}\left(U F_{j} V\right)^{*} A_{i}\left(U F_{j} V\right), \quad i=1, \ldots, k,
$$

Furthermore,

(1) $\Phi$ in (a) is unital if and only if $D$ in (b) can be chosen to be column stochastic. 
(2) $\Phi$ in (a) is trace preserving if and only if $D$ in (b) can be chosen to be row stochastic.

(3) $\Phi$ in (a) is unital and trace preserving if and only if $D$ in (b) can be chosen to be doubly stochastic.

Proof. By Proposition 1.4, we may assume that $A_{i}=\operatorname{diag}\left(\mathbf{a}_{i}\right)$ and $B_{i}=\operatorname{diag}\left(\mathbf{b}_{i}\right)$ for $i=1, \ldots, k$, and take $U=I_{n}, V=I_{m}$ in $(2.1)$.

(a) $\Rightarrow$ (b): Suppose there is a completely positive map $\Phi: M_{n} \rightarrow M_{m}$ such that $\Phi\left(A_{i}\right)=B_{i}$ for $i=1, \ldots, k$. By $(1.1)$, we have $F^{j}=\left(f_{p q}^{j}\right) \in M_{n, m}, j=1, \ldots, r$, such that

$$
\Phi(X)=\sum_{j=1}^{r} F_{j}^{*} X F_{j} .
$$

For $1 \leq p \leq n$ and $1 \leq q \leq m$, let $d_{p q}=\sum_{j=1}^{r}\left|f_{p q}^{j}\right|^{2}$. Then $D=\left(d_{p q}\right)$ is an $n \times m$ nonnegative matrix such that

$$
\left(b_{i j}\right)=\left(a_{i j}\right) D .
$$

(b) $\Rightarrow\left(\right.$ a): Suppose $D=\left(d_{p q}\right)$ is a nonnegative matrix satisfying $\left(b_{i j}\right)=\left(a_{i j}\right) D$. Let $F_{j}$ be defined as in the theorem. Then direct computation shows that for every $\mathbf{x}=\left(x_{1}, \ldots, x_{n}\right)$, $\sum_{j=1}^{r} F_{j}^{*} \operatorname{diag}(\mathbf{x}) F_{j}$ is a diagonal matrix with diagonal $\mathbf{y}=\mathbf{x} D$. Hence, for $i=1, \ldots, k$, we have

$$
\operatorname{diag}\left(\mathbf{b}_{i}\right)=\sum_{j=1}^{m} F_{j}^{*} \operatorname{diag}\left(\mathbf{a}_{i}\right) F_{j},
$$

for $i=1, \ldots, k$.

For $N \geq 1$, let $\mathbf{1}_{N}$ be a row vector of $N 1$ 's, then we have

$\Phi$ is unital $\Leftrightarrow \Phi\left(I_{n}\right)=I_{m} \Leftrightarrow \mathbf{1}_{m}=\mathbf{1}_{n} D \Leftrightarrow D$ is column stochastic.

This proves (1). The proof for cases (2) and (3) are similar.

Condition (b) in Theorem 2.1 has been studied in $[5,17,1]$ with row (column, doubly, respectively) $D$. An analog of (3) for $I I_{1}$ factor is also proven in [1].

A completely positive map $\Phi: M_{n} \rightarrow M_{n}$ is called mixed unitary if there exist unitary matrices $U_{1}, \ldots, U_{r} \in M_{n}$ and positive numbers $t_{1}, \ldots, t_{r}$ summing up to 1 such that

$$
\Phi(X)=\sum_{j=1}^{r} t_{j} U_{j}^{*} X U_{j} .
$$

Clearly, every mixed unitary completely positive map is unital and trace preserving. For $n \geq 3$, there exists [11] a unital trace preserving completely positive map which is not mixed unitary.

By the Birkhoff Theorem [13], a doubly stochastic matrix $D$ can be expressed in the form

$$
D=\sum_{j=1}^{r} t_{j} P_{j}
$$

for some positive numbers $t_{1}, \ldots, t_{r}$ summing up to 1 and permutation matrices $P_{1}, \ldots, P_{r} \in M_{n}$. Using this result and (3) in Theorem 2.1, we have the following corollary. 
Corollary 2.2. Under the hypothesis of Theorem 2.1, suppose there is a unital trace preserving completely positive map $\Phi$ satisfying (a), and $D$ is a doubly stochastic matrix satisfying (b). Let $D$ be expressed as in (2.2). Then the mixed unitary map $\Psi: M_{n} \rightarrow M_{n}$ defined by

$$
\Psi(X)=\sum_{j=1}^{r} t_{j}\left(U P_{j} V\right)^{*} X\left(U P_{j} V\right)
$$

also satisfies $\Psi\left(A_{i}\right)=B_{i}$ for $i=1, \ldots, k$.

Remark 2.3. The following remarks concerning Theorem 2.1 are in order.

a) In Theorem 2.1, if the matrices $U$ and $V$ in the hypothesis are merely invertible instead of unitary, conditions (a) and (b) are still equivalent. In other words, the result applies to two families $\left\{A_{1}, \ldots, A_{k}\right\} \subseteq H_{n}$ and $\left\{B_{1}, \ldots, B_{k}\right\} \subseteq H_{m}$ such that each family is simultaneously congruent to diagonal matrices.

b) To check conditions (b) and the corresponding ones in (1), (2) and (3), one can use standard linear programming techniques.

To check condition (1), one can divide the problem of finding a column stochastic $D$ into $m$ independent problems of determining the columns $\mathbf{d}_{1}, \ldots, \mathbf{d}_{m}$ of $D$ as follows.

Set $A=\left(a_{i j}\right)_{1 \leq i \leq k, 1 \leq j \leq n}$. For each $q=1, \ldots, m$, determine a nonnegative vector $\mathbf{d}_{q} \in \mathbb{R}^{n}$ with entries summing up to one such that $A \mathbf{d}_{q}=\left(b_{1 q}, \ldots, b_{k q}\right)^{t}$.

Clearly, the solution set of each of the problems is a convex polyhedron, namely,

$$
P_{q}=\left\{\mathbf{d}=\left[\begin{array}{c}
d_{1 q} \\
\vdots \\
d_{n q}
\end{array}\right]: d_{p q} \geq 0, \sum_{p=1}^{n} d_{p q}=1, \quad \text { and } A \mathbf{d}=\left[\begin{array}{c}
b_{1 q} \\
\vdots \\
b_{k q}
\end{array}\right]\right\}
$$

in $\mathbb{R}^{n}$. By standard convex analysis, if $A$ has rank $k$, then the extreme points of the polyhedron $P_{q}$, if non-empty, has at most $k+1$ nonzero entries. Thus, one can construct a sparse matrix $D$ as a solution using the extreme points in the solution sets $P_{q}$.

To check condition (2), one can construct an extreme point (with sparse pattern) of the set of row stochastic matrices $D$ satisfying $\left(b_{i j}\right)=\left(a_{i j}\right) D$. However, unlike the checking of (1), one cannot treat individual rows separately to reduce the complexity of the computation.

c) In the construction of $F_{1}, \ldots, F_{r}$ in the last assertion of the theorem, we see that each $F_{j}$ has rank at most $\ell=\min \{m, n\}$ so that the corresponding completely positive map $\Phi$ is a super $\ell$-positive map [18].

d) Note that in our construction, if $A_{1}, \ldots, A_{k}$ and $B_{1}, \ldots, B_{k}$ are in diagonal form, then the map $A_{i} \mapsto F_{j}^{*} A_{i} F_{j}$ has only one nonzero entry at the $(j, j)$ position obtained by taking a nonnegative combination of the diagonal entries of $A_{i}$. In the context of quantum information science, it is easy to implement the map (quantum operation) $\Phi$ on diagonal matrices because all the actions only take place at the diagonal entries (classical channels). 


\section{Completely positive Maps on A Single matrix}

For a single matrix, we can give a more detailed analysis of the result in Theorem 2.1, and show that the study is related to other topics such as eigenvalue inequalities. Moreover, the results show that there are some results on trace preserving completely positive maps with no analogs for unital completely positive maps, and vice versa.

\subsection{Unital completely positive maps.}

Theorem 3.1. Suppose $A \in H_{n}$ and $B \in H_{m}$ have eigenvalues $a_{1}, \ldots, a_{n}$ and $b_{1}, \ldots, b_{m}$, respectively. Let $\mathbf{a}=\left(a_{1}, \ldots, a_{n}\right)$ and $\mathbf{b}=\left(b_{1}, \ldots, b_{m}\right)$. The following conditions are equivalent.

(a) There is a (unital) completely positive map $\Phi: M_{n} \rightarrow M_{m}$ such that $\Phi(A)=B$.

(b) There is a nonnegative (column stochastic) matrix $D=\left(d_{p q}\right)$ such that $\mathbf{b}=\mathbf{a} D$.

(c) There are real numbers $\gamma_{1}, \gamma_{2} \geq 0$ (with $\gamma_{1}=\gamma_{2}=1$ ) such that

$$
\gamma_{2} \min \left\{a_{i}: 1 \leq i \leq n\right\} \leq b_{j} \leq \gamma_{1} \max \left\{a_{i}: 1 \leq i \leq n\right\} .
$$

for all $1 \leq j \leq m$.

Proof. By Theorem 2.1, (a) and (b) are equivalent. Without loss of generality, we may assume that $a_{1} \geq \cdots \geq a_{n}$ and $b_{1} \geq \cdots \geq b_{m}$.

(b) $\Rightarrow$ (c): Suppose there is a nonnegative matrix $D=\left(d_{p q}\right)$ such that $\mathbf{b}=\mathbf{a} D$. Let $\gamma_{1}=\sum_{p=1}^{n} d_{p 1}$ and $\gamma_{2}=\sum_{p=1}^{n} d_{p m}$. Then for each $1 \leq j \leq m$, we have

$$
\gamma_{2} a_{n}=\sum_{p=1}^{n} d_{p m} a_{n} \leq \sum_{p=1}^{n} d_{p m} a_{p}=b_{m} \leq b_{j} \leq b_{1}=\sum_{p=1}^{n} d_{p 1} a_{p} \leq \sum_{p=1}^{n} d_{p 1} a_{1}=\gamma_{1} a_{1} .
$$

If $D$ is column stochastic, then it follows from definition that $\gamma_{1}=\gamma_{2}=1$.

(c) $\Rightarrow$ (b): Suppose (c) holds. Then for each $i=1, \ldots m$, there exists $0 \leq t_{i} \leq 1$ such that $b_{i}=t_{i} \gamma_{1} a_{1}+\left(1-t_{i}\right) \gamma_{2} a_{n}$. Let $D$ be the $n \times m$ matrix

$$
\left[\begin{array}{cccc}
t_{1} \gamma_{1} & t_{2} \gamma_{1} & \cdots & t_{m} \gamma_{1} \\
0 & 0 & \cdots & 0 \\
\vdots & \vdots & \vdots & \vdots \\
0 & 0 & \cdots & 0 \\
\left(1-t_{1}\right) \gamma_{2} & \left(1-t_{2}\right) \gamma_{2} & \cdots & \left(1-t_{m}\right) \gamma_{2}
\end{array}\right]
$$

Then $\mathbf{b}=\mathbf{a} D$ and $D$ is column stochastic if $\gamma_{1}=\gamma_{2}=1$.

Corollary 3.2. If $A \in H_{n}$ and $B \in H_{m}$ are nonzero positive semi-definite, then there is a completely positive map $\Phi: M_{n} \rightarrow M_{m}$ such that $\Phi(A)=B$, and there is a completely positive map $\Psi: M_{m} \rightarrow M_{n}$ such that $\Psi(B)=A$.

Using Theorem 3.1, one can construct a pair of density matrices $(A, B) \in H_{n} \times H_{m}$ such that there is a unital completely positive map $\Phi$ such that $\Phi(A)=B$, but there is no unital completely positive map $\Psi$ such that $\Psi(B)=A$. 


\subsection{Trace preserving completely positive maps.}

Theorem 3.3. Suppose $A \in H_{n}$ and $B \in H_{m}$ have eigenvalues $a_{1}, \ldots, a_{n}$ and $b_{1}, \ldots, b_{m}$ respectively. Let $\mathbf{a}=\left(a_{1}, \ldots, a_{n}\right)$ and $\mathbf{b}=\left(b_{1}, \ldots, b_{m}\right)$. The following conditions are equivalent.

(a) There is a trace preserving completely positive map $\Phi: M_{n} \rightarrow M_{m}$ such that $\Phi(A)=B$.

(b) There exists an $n \times m$ row stochastic matrix $D$ such that $\mathbf{b}=\mathbf{a} D$. Moreover, we can assume that the $p$ th and $q$ th row are identical whenever $a_{p} a_{q}>0$, and the $p$ th row of $D$ can be arbitrary (nonnegative with entries summing up to 1) if $a_{p}=0$.

(c) We have $\operatorname{tr} A=\operatorname{tr} B$ and $\sum_{p=1}^{n}\left|a_{p}\right| \geq \sum_{q=1}^{m}\left|b_{q}\right|$. Equivalently, $\operatorname{tr} A=\operatorname{tr} B$ and the sum of the positive (negative) eigenvalues of $A$ is not smaller (not larger) than the sum of the positive (negative) eigenvalues of $B$.

Proof. For simplicity, we assume that $a_{1} \geq \cdots a_{r} \geq 0>a_{r+1} \geq \cdots \geq a_{n}$ and $b_{1} \geq \cdots \geq b_{s} \geq$ $0>b_{s+1} \geq \cdots \geq b_{m}$. Let $a_{+}=\sum_{p=1}^{r} a_{p}, a_{-}=\sum_{p=r+1}^{n} a_{p}$ and $b_{+}=\sum_{q=1}^{s} b_{q}, b_{-}=\sum_{q=s+1}^{m} b_{q}$.

By Theorem 2.1, we have (b) $\Rightarrow$ (a). Also, by Theorem 2.1, if (a) holds, then $\mathbf{b}=\mathbf{a} D$ for an $n \times m$ row stochastic matrix $D$. Next, we show that $D$ can be chosen to satisfy the second assertion of condition (b). To this end, let $\mathbf{d}_{1}, \ldots, \mathbf{d}_{n}$ be the rows of $D$. Clearly, if $a_{p}=0$, we can replace the $p$ th row of $D$ by any nonnegative vectors with entries summing up to 1 to get $\tilde{D}$ and we still have $\mathbf{a} \tilde{D}=\mathbf{a} D=\mathbf{b}$. Now, suppose $a_{1}>0$. Then $a_{+}>0$. We can replace the first $r$ rows (or the rows correspond to $a_{p}>0$ ) by

$$
\mathbf{d}_{+}=\sum_{j=1}^{r} \frac{a_{j}}{a_{+}} \mathbf{d}_{j}
$$

to obtain $\tilde{D}$. Then $\mathbf{d}_{+}$has nonnegative entries summing up to 1 , and $\mathbf{a} \tilde{D}=\mathbf{a} D=\mathbf{b}$. Similarly, suppose $a_{n}<0$. Then $a_{-}<0$. We can further replace the last $n-s$ row of $D$ by

$$
\mathbf{d}_{-}=\sum_{j=s+1}^{n} \frac{a_{j}}{a_{-}} \mathbf{d}_{j}
$$

to obtain $\tilde{D}$. Then $\mathbf{d}_{-}$has nonzero entries summing up to 1 , and $\mathbf{a} \tilde{D}=\mathbf{a} D=\mathbf{b}$.

(b) $\Rightarrow$ (c): Suppose $\mathbf{b}=\mathbf{a} D$. We have

$$
\operatorname{tr} B=\sum_{q=1}^{m} b_{q}=\sum_{q=1}^{m} \sum_{p=1}^{n} a_{p} d_{p q}=\sum_{p=1}^{n} a_{p}\left(\sum_{q=1}^{m} d_{p q}\right)=\sum_{p=1}^{n} a_{p}=\operatorname{tr} A
$$

and

$$
\sum_{q=1}^{m}\left|b_{q}\right|=\sum_{q=1}^{m}\left|\sum_{p=1}^{n} a_{p} d_{p q}\right| \leq \sum_{q=1}^{m} \sum_{p=1}^{n}\left|a_{p}\right| d_{p q}=\sum_{p=1}^{n}\left|a_{p}\right|\left(\sum_{q=1}^{m} d_{p q}\right)=\sum_{p=1}^{n}\left|a_{p}\right|
$$

Since

$$
\sum_{i=1}^{n} a_{i}=\sum_{j=1}^{m} b_{j}, \quad \sum_{i=1}^{n}\left|a_{i}\right|=a_{+}-a_{-}=\sum_{i=1}^{n} a_{i}-2 a_{-}=2 a_{+}-\sum_{i=1}^{n} a_{i},
$$


and

$$
\sum_{j=1}^{m}\left|b_{j}\right|=b_{+}-b_{-}=\sum_{j=1}^{m} b_{j}-2 b_{-}=2 b_{+}-\sum_{j=1}^{m} b_{j},
$$

the last assertion of (c) follows.

(c) $\Rightarrow$ (b): Suppose $\operatorname{tr} A=\operatorname{tr} B, a_{+} \geq b_{+}$and $a_{-} \leq b_{-}$. Let

$$
t_{q}= \begin{cases}\frac{b_{q}}{a_{+}} & \text {for } 1 \leq q \leq s \\ \frac{b_{q}}{a_{-}} & \text {for } s<q \leq m .\end{cases}
$$

Here, if $a_{+}=0$ then $b_{+}=0$, and we can set $t_{q}=0$ for $1 \leq q \leq s$. If $a_{-}=0$ then $b_{-}=0$, and $s=m$. Therefore, $t_{q} \geq 0$ for all $1 \leq q \leq m$. We have

$$
a_{+} \geq b_{+}=\left(a_{+}\right) \sum_{q=1}^{s} t_{q}, \quad \text { and } \quad\left|a_{-}\right| \geq\left|b_{-}\right|=\left|a_{-}\right| \sum_{q=s+1}^{n} t_{q} .
$$

Let $u=1-\sum_{q=1}^{s} t_{q} \geq 0, v=1-\sum_{q=s+1}^{n} t_{q} \geq 0$ and $D$ be an $n \times m$ row stochastic matrix with

$$
p \text { th row }= \begin{cases}\left(t_{1}, t_{2}, \ldots, t_{s}, 0, \ldots, 0, u\right) & \text { for } 1 \leq p \leq r \\ \left(0, \ldots, 0, t_{s+1}, t_{s+2}, \ldots, t_{m-1}, t_{m}+v\right) & \text { for } r+1 \leq p \leq n\end{cases}
$$

Since

$$
u a_{+}+v a_{-}=\left(a_{+}-b_{+}\right)+\left(a_{-}-b_{-}\right)=0
$$

we have $\mathbf{b}=\mathbf{a} D$.

Corollary 3.4. If $A \in H_{n}$ and $B \in H_{m}$ are density matrices, i.e. positive semi-definite and $\operatorname{tr} A=\operatorname{tr} B=1$, then there is a completely positive map $\Phi: M_{n} \rightarrow M_{m}$ such that $\Phi(A)=B$, and there is a completely positive map $\Psi: M_{m} \rightarrow M_{n}$ such that $\Psi(B)=A$.

As remarked after Corollary 3.2, one may not be able to find a unital completely positive map taking a density matrix $B \in H_{m}$ to another density matrix $A \in H_{n}$ even if there is a unital positive completely positive map sending $A$ to $B$.

\subsection{Unital trace preserving completely positive maps.}

Suppose there is a unital completely positive map sending $A$ to $B$, and also a trace preserving completely positive map sending $A$ to $B$. Is there a unital trace preserving completely positive map sending $A$ to $B$ ? If the answer were "yes", then one can reduce a complicated problem into two relatively simple problems. Unfortunately, the following example shows that the answer is negative.

Example 3.5. Suppose $A=\operatorname{diag}(4,1,1,0)$ and $B=\operatorname{diag}(3,3,0,0)$. By Theorems 3.1 and 3.3 there is a trace preserving completely positive map sending $A$ to $B$, and also a unital completely positive map sending $A$ to $B$. Let $A_{1}=A-I_{4}=\operatorname{diag}(3,0,0,-1)$ and $B_{1}=$ 
$B-I_{4}=\operatorname{diag}(2,2,-1,-1)$. By Theorem 3.3 , there is no trace preserving completely positive map sending $A_{1}$ to $B_{1}$. Hence, there is no unital trace preserving completely positive map sending $A$ to $B$.

As shown in Corollary 2.2, if there is a unital trace preserving map sending a commuting family in $H_{n}$ to a commuting family in $H_{m}$, then we may chose the map to be mixed unitary. In the following, we show that for the case when $k=1$ in Theorem 2.1, one can even assume that the map is the average of $n$ unitary similarity transforms.

Let $\mathbf{a}=\left(a_{1}, \ldots, a_{n}\right), \mathbf{b}=\left(b_{1}, \ldots, b_{n}\right) \in \mathbb{R}^{n}$. We say that $\mathbf{b}$ is majorized by $\mathbf{a}(\mathbf{b} \prec \mathbf{a})$ if for every $1 \leq k<n$, the sum of the $k$ largest entries of $\mathbf{b}$ is less than or equal to the sum of the $k$ largest entries of $\mathbf{a}$, and $\sum_{i=1}^{n} a_{i}=\sum_{i=1}^{n} b_{i}$.

Theorem 3.6. Suppose $A \in H_{n}$ and $B \in H_{m}$ have eigenvalues $a_{1}, \ldots, a_{n}$ and $b_{1}, \ldots, b_{m} r e-$ spectively. Let $\mathbf{a}=\left(a_{1}, \ldots, a_{n}\right)$ and $\mathbf{b}=\left(b_{1}, \ldots, b_{m}\right)$. The following are equivalent.

(a) There exists a unital trace preserving completely positive map $\Phi$ such that $\Phi(A)=B$.

(a1) There exists a mixed unitary completely positive map $\Phi$ such that $\Phi(A)=B$.

(a2) There exist unitary matrices $U_{1}, \ldots, U_{n} \in M_{n}$ such that $B=\frac{1}{n} \sum_{j=1}^{n} U_{j}^{*} A U_{j}$.

(a3) For each $t \in \mathbb{R}$, there exists a trace preserving completely positive map $\Phi_{t}$ such that $\Phi_{t}(A-t I)=B-t I$.

(b) There is a doubly stochastic matrix $D$ such that $\mathbf{b}=\mathbf{a} D$.

(b1) There is a unitary matrix $W \in M_{n}$ such that $W \operatorname{diag}\left(a_{1}, \ldots, a_{n}\right) W^{*}$ has diagonal entries $b_{1}, \ldots, b_{n}$.

(c) $\mathbf{b} \prec \mathbf{a}$.

Moreover, if condition (b) holds and $D=\sum_{\ell=1}^{r} t_{\ell} P_{\ell}$ such that $t_{1}, \ldots, t_{r}$ are positive numbers summing up to 1 and $P_{1}, \ldots, P_{r}$ are permutation matrices, then $B=\sum_{j=1}^{r} V^{*} P_{j}^{t} U^{*} A U P_{j} V$, where $U^{*} A U=\operatorname{diag}\left(a_{1}, \ldots, a_{n}\right)$ and $V^{*} B V=\operatorname{diag}\left(b_{1}, \ldots, b_{n}\right)$.

Proof. (b) $\Longleftrightarrow$ (c) is a standard result of majorization; see [13].

The implications (a2) $\Rightarrow(\mathrm{a} 1) \Rightarrow$ (a) $\Rightarrow$ (a3) are obvious.

(a3) $\Rightarrow$ (c) : We may assume that $a_{1} \geq \cdots \geq a_{n}$ and $b_{1} \geq \cdots \geq b_{n}$. For $1 \leq k<n$ choose $t$ such that $a_{k} \geq t \geq a_{k+1}$. Then there is a trace preserving completely positive linear map $\Phi_{t}$ such that $\Phi_{t}\left(A-t I_{n}\right)=B-t I_{n}$. By Theorem 3.3, the sum of the $k$ positive eigenvalues of $B-t I_{n}$ is no larger than that of $A-t I_{n}$. Thus,

$$
\sum_{i=1}^{k} b_{i}-k t=\sum_{i=1}^{k}\left(b_{i}-t\right) \leq \sum_{i=1}^{k}\left(a_{i}-t\right)=\sum_{i=1}^{k} a_{i}-k t
$$

We see that $\sum_{i=1}^{k} b_{i} \leq \sum_{i=1}^{k} a_{i}$ for $k=1, \ldots, n-1$. Since $\operatorname{tr} A=\operatorname{tr} B$, we have $\mathbf{b} \prec \mathbf{a}$.

(c) $\Rightarrow$ (b1) is a result of Horn [8]. 
(b1) $\Rightarrow\left(\right.$ a2) : Suppose $W$ is a unitary matrix such that the diagonal of $W \operatorname{diag}\left(a_{1}, \ldots, a_{n}\right) W^{*}$ has diagonal entries $b_{1}, \ldots, b_{n}$. Let $w=e^{i 2 \pi / n}, P=\operatorname{diag}\left(1, w, \ldots, w^{n-1}\right)$. Then

$$
B=\frac{1}{n} \sum_{j=1}^{n} U W\left(P^{j}\right)^{*} W U A U^{*} W^{*}\left(P^{j}\right) U^{*}
$$

Thus, (a2) holds.

In the context of quantum information theory, a completely positive map in condition (a1) of the above theorem is a mixed unitary quantum channel/operation. By the above theorem, the existence of a mixed unitary quantum channel $\Phi$ taking a quantum state $A \in H_{n}$ to a quantum state $B \in H_{m}$ can be described in terms of trace preserving completely positive maps, namely, condition (a3). However, despite the duality of the two classes of maps, there is no analogous condition in terms of unital completely positive map; see Proposition 1.2.

\section{Additional Remarks and future Research}

To study unital completely positive maps connecting two families $\left\{A_{1}, \ldots, A_{k}\right\} \subseteq H_{n}$ and $\left\{B_{1}, \ldots, B_{k}\right\} \subseteq H_{m}$, one can use the results on completely positive maps and add $I_{n}$ and $I_{m}$ to the two families.

The following result shows that the study of a completely positive maps sending $A_{1}, \ldots, A_{k} \in$ $H_{n}$ to $B_{1}, \ldots, B_{k} \in H_{m}$ can be reduced to the study of unital completely positive maps.

Theorem 4.1. Let $A_{1}, \ldots, A_{k} \in H_{n}$, and $B_{1}, \ldots, B_{k} \in H_{m}$. There is a completely positive map $\Phi: M_{n} \rightarrow M_{m}$ such that $\Phi\left(A_{j}\right)=B_{j}$ for $j=1, \ldots, k$ if and only if there exists $\gamma>0$ and a unital completely positive map $\Psi: M_{n+1} \rightarrow M_{m}$ such that $\Psi\left(A_{j} \oplus[0]\right)=\gamma^{-1} B_{j}$ for $j=1, \ldots, k$.

Proof. Suppose $\Phi: M_{n} \rightarrow M_{m}$ has operator sum representation (1.1) and satisfies $\Phi\left(A_{j}\right)=B_{j}$ for $j=1, \ldots, k$. Let $\Phi\left(I_{n}\right)=P \in H_{m}$. Choose $\gamma>0$ such that $\gamma I_{m}-P$ is positive semidefinite. Then we have $\gamma I_{m}-P=\sum_{j=1}^{s} g_{j}^{*} g_{j}$ for some $1 \times m$ matrices $g_{j}$. For $j=1, \ldots, \tilde{r}$ with $\tilde{r}=\max \{r, s\}$, let $\tilde{F}_{j} \in M_{n+1, m}$ be such that $\tilde{F}_{j}=\left[\begin{array}{c}F_{j} \\ g_{j}\end{array}\right]$, where $F_{j}=0$ if $j>r$ and $g_{j}=0$ if $j>s$. Define $\Psi: M_{n+1} \rightarrow M_{m}$ by $\Psi(X)=\frac{1}{\gamma} \sum_{j=1}^{\tilde{r}} \tilde{F}_{j}^{*} X \tilde{F}_{j}$. One readily checks that $\Psi\left(I_{n+1}\right)=I_{m}$ and $\Psi\left(A_{j} \oplus[0]\right)=\gamma^{-1} B_{j}$ for $j=1, \ldots, k$.

Conversely, suppose $\gamma>0$ and $\Psi: M_{n+1} \rightarrow M_{m}$ is a unital completely positive map such that $\Psi\left(A_{j} \oplus[0]\right)=\gamma^{-1} B_{j}$ for $j=1, \ldots, k$, then one can check $\Phi: M_{n} \rightarrow M_{m}$ defined by $\Phi(X)=\gamma \Psi(X \oplus[0])$ is a completely positive map satisfying $\Phi\left(A_{j}\right)=B_{j}$ for $j=1, \ldots, k$.

Finding a unital completely positive map connecting two general (non-commuting) families of Hermitian matrices is very challenging. In the case of non-commuting families $\left\{A_{1}, A_{2}\right\} \subset H_{n}$, and $\left\{B_{1}, B_{2}\right\} \subseteq H_{m}$ with two elements, the problems reduce to the study of unital completely positive maps $\Phi$ satisfying $\Phi\left(A_{1}+i A_{2}\right)=B_{1}+i B_{2}$. For $n=2,3$. There are partial answers of 
the problem in terms of the numerical range and dilation of operators. Recall that the numerical range of $T \in M_{n}$ is the set

$$
W(T)=\left\{x^{*} T x: x \in \mathbb{C}^{n}, x^{*} x=1\right\},
$$

and $T$ has a dilation $\tilde{T} \in M_{m}$ if there is an $n \times m$ matrix $X$ such that $X X^{*}=I_{n}$ and $X \tilde{T} X^{*}=T$. We have the following result; see $[3,4]$.

Theorem 4.2. Let $(A, B) \in M_{n} \times M_{m}$. Suppose $n=2$, or $n=3$ such that $A$ is unitarily reducible, i.e., $A$ is untiarily similar to $A_{1} \oplus[\alpha]$ for some $A_{1} \in M_{2}$ and $\alpha \in \mathbb{C}$. Then the following conditions are equivalent.

(a) There is a unital completely positive map $\Phi: M_{n} \rightarrow M_{m}$ such that $\Phi(A)=B$.

(b) $B$ has a dilation of the form $I_{r} \otimes A$.

(c) $W(B) \subseteq W(A)$.

Special cases of the above theorem include the case when $A \in M_{3}$ is a normal matrix. However, there are examples showing that the result fails if $A$ is an arbitrary matrix in $M_{3}$ or an arbitrary normal matrix in $M_{4}$; see [3].

In connection to Theorem 4.2, one may ask for the condition of $A \in M_{n}$ to be a dilation of $B \in M_{m}$ itself. The problem is challenging even for normal matrices $A$ and $B$; see [9].

Also, it is interesting to impose condition on the Kraus (Choi) rank, i.e., the minimum number of matrices $F_{1}, \ldots, F_{r}$ needed in the operator sum representation of the completely positive maps. As mentioned in Proposition 1.3, the study is related to the study of principal submatrices of a Hermitian matrices, which is related to the study of spectral inequalities and LittlewoodRichardson rule; see $[6,12]$.

\section{Acknowledgment}

Part of the results in the paper was reported at the Workshop on Mathematics in Experimental Quantum Information Processing, IQC, Waterloo, August 10-14, 2009. The comments of the participants and the support of the organizer are graciously acknowledged.

Research of both authors are supported by USA NSF. The first author was also supported by a HK RCG grant, and the Key Disciplines of Shanghai Municipality Grant S30104. He is an honorary professor of the University of Hong Kong, the Taiyuan University of Technology, and the Shanghai University. The paper was done while he was taking his SSRL leave from the College of William and Mary and visiting the George Washington University in the fall of 2010.

\section{REFERENCES}

1. M. Argerami and P. Massey (2008). The local form of doubly stochastic maps and joint majorization in $\mathrm{II}_{1}$ factors. Integral Equations Operator Theory., 61, 119.

2. M.D. Choi (1975). Completely positive linear maps on complex matrices. Linear Algebra and Appl., 10, 285-290.

3. M.D. Choi and C.K. Li (2000). Numerical ranges and dilations. Linear and Multilinear Algebra, 47, 35-48. 
4. M.D. Choi and C.K. Li (2001). Constrained unitary dilations and numerical ranges. J. Operator Theory, 46, 435-447.

5. G. Dahl(1999). Matrix majorization. Linear Algebra Appl., 288, 53-73.

6. S. Formin, W. Fulton, C.K. Li and Y.T. Poon (2005). Eigenvalues, singular values, and LittlewoodRichardson Coefficients. Amer. J. Math., 127, 101-127.

7. W. Fulton (2000). Eigenvalues of majorized Hermitian matrices, and Littlewood-Richardson coefficients. Linear Algebra Appl., 319, 23-36.

8. A. Horn (1954). Doubly stochastic matrices and the diagonal of a rotation matrix. Amer. J. Math., 76, 620-630.

9. J. Holbrook (2008). Private communication.

10. K. Kraus(1983). States, effects, and operations: fundamental notions of quantum theory, 190, Lectures in mathematical physics at the University of Texas at Austin, Lecture Notes in Physics, Springer-Verlag, Berlin-Heidelberg.

11. L. Landau and R. Streater (1993). On Birkhoffs theorem for doubly stochastic completely positive maps of matrix algebras. Linear Algebra and Appl. , 193, 193 107-127.

12. C.K. Li and Y. T. Poon (2003). Principal Submatrices of a Hermitian matrix. Linear and Multilinear Algebra, 51, 199-208.

13. A.W. Marshall and I. Olkin (1979). Inequalities: The Theory of Majorizations and Its Applications. Academic Press.

14. M.A. Nielsen and I.L. Chuang (2000). Quantum Computation and Quantum information. Cambridge University Press.

15. V.I. Paulsen (1986). Completely Bounded Maps and Dilations. Pitman Research Notes in Mathematical Series.

16. D. Petz (2008). Quantum Information Theory and Quantum Statistics.Springer-Verlag, Heidelberg.

17. F.D.M. Pería, P.G. Massey and L.E. Silvestre (2005). Weak Matrix Majorization, Linear Algebra Appl., 403, 343-368.

18. Ł. Skowronek, E. Størmer, K. Życzkowski (2009). Cones of positive maps and their duality relations, J. Math. Phys., 50, 062106.

19. W.F. Stinespring (1955). Positive functions on $C^{*}$-algebras, Proc. Amer. Math. Soc., 6, 211-216.

Department of Mathematics, College of William \& Mary, Williamsburg, VA 23187

E-mail address: ckli@math.wm.edu

Department of Mathematics, Iowa State University, Ames, IA 50051

E-mail address: ytpoon@iastate.edu 